cortex. (Ann Neuro 1983; 14:471). Motor dysfunction generally deteriorates steadily in Rett syndrame and parkinson-like features predominate during adolescence and early adulthood, suggesting a progressive involvement of the nigrostriatal system. Jellinger K, Percy AK et al. (Acta Neuropathologica 1988; $76: 142$ ) have described the autopsy findings in nine girls with Rett syndrome, ages 4-17 years. All brains were smaller than normal, lipofuscin was deposited in neuronal cytoplasm, melanin was absent in substantia nigra, and indications of dopaminergic nigrostriatal dysfunction were suggested. The intensive search for a biological marker for Rett syndrome continues.

\title{
RETT SYNDROME AND HELLER DEMENTIA
}

Six girls with Rett syndrome and two boys with Heller dementia are reported and contrasted with children with classic autism fram the Department of Neuroscience and Pediatrics, University of North Dakota School of Medicine, Grand Forks, North Dakota, and the Kennedy Institute, Johns Hopkins Medical Institutions, Baltimore, MD. The study was performed in response to a report that Ret $t$ syndrome may be a form of Heller dementia with a predilection for girls (Millichap JG. Lancet Feb 21, 1987; 1:440). All eight children differed from those with classic autism in that they had normal prenatal and perinatal periods, followed by marked developmental regression, after which they acquired few or no skills. The boys with a diagnosis of Heller dementia differed from the girls with Rett syndrome in terms of estimated prevalence, age at onset, stereotypic breathing patterns, midline hand stereotypies, hand and gait apraxia, and speech development. The authors found no stereotyped movements in their two patients with Heller dementia although these have been described in other studies. The patients showed similarities in the normal prenatal and perinatal periods, behavioral, social and psychomotor regression, and epilepsy. The authors suggested that these children should be distinguished from those with classic autism and should be classified as "pervasive disintegrative disorder, Heller type" and "pervasive disintegrative disorder, Rett type". (Burd L, Fisher W, Kerbeshian, J. Pervasive disintegrative disorder: Are Rett syndrome and Heller dementia infantilis subtypes? Dev Med Child Neurol October 1989; $\underline{31}: 609-616)$.

COMNENT. In 1908 , almost 60 years before the first description of Rett syndrome, Heller reported an infantile dementia with symptams and a course similar in some respects to that of Rett syndrome. By 1930, Heller had collected 28 cases of dementia in young children who previously had been entirely normal in development. Wi thout antecedent illness, a change in mood and behavior was noticed. The children became irritable, negativistic, and disobedient; they had outbursts of temper without provocation; they showed signs of anxiety; and a mental regression led to a complete loss of speech and deterioration within a few months. Motor restlessness and stereotyped 
repetitive movements and mannerisms with grimacing and tics were most conspicuous. Seizures and growth retardation were mentioned in later reports. The cause was unknown but a suspected organic lesion was confirmed in cases examined at necropsy. Diffuse lipoid cell degeneration of the cortical neurons, atrophy of the brain, small disorganized cortical neurons lacking in Nissl bodies, and marginal gliosis have been described, and abnormalities in plasma lipids. Heller dementia is recognized as a syndrome in text books of pediatric neurology and child psychiatry although some have suggested that some examples of the syndrome may have been confused with childhood schizophrenia, and other degenerative brain diseases. Heller dementia occurs in both boys and girls and unlike Rett syndrome, the disorder was not restricted to girls. It is surprising that in publications on Rett syndrome Heller dementia seems to have been overlooked in the differential diagnosis. Drs. Rett and Olsson have now corrected this omission (Dev Med Child Neurol 1987; 29:834) but believe that research wil 1 show Ret syndrome to be an independent disorder. They admit that the differentiation may be difficult in older children and that the range of variations of both syndromes cannot be determined until the etiology is known. The suggestion that most cases of Heller dementia were disintegrative psychoses (Stephenson JBP, Kerr AM. Lancet March $28,1987 ; 1: 741)$ does not fit with published neuropathological findings, and the introduction of the term "pervasive disintegrative disorder, Heller type and Rett type", based on two patients in the present report, is probably not of value in the classification of these dementias.

\section{NETROCUTANEOUS DISORDERS}

\section{LEOPARD SYNDROME}

A 12 year old boy with multiple lentigines (Leopard) syndrome in association with Gerstmann syndrame and CT abnormalities is reported from the Department of Pediatrics and Pediatric Neurology, Beilinson Medical Center, Petah Tikva and Tel Aviv University, Sackler School of Medicine, Petah Tikva, Israel. Learning difficulties were first observed at five years of age. He had a single simple febrile convulsion at three years of age. Several hundred hyperpigmented skin lesions over the face, trunk, and extremities had appeared gradually after birth and had increased in number and size. Mild dysmorphic features included hypertelorism, epicanthal folds, cubitus valgus, and pterygium colli. A systolic cardiac murmur was indicative of pulmonic stenosis and was accompanied by EKG changes. The signs of Gerstmann syndrome included dyscalculia, left/right disorientation, finger agnosia, and dysgraphia. His IQ score was 86. Cranial CT showed dilatation of the left lateral ventricle especially in the occipital horn and mild atrophy of the left parietal lobe. (Garty B-Z et al. Gertsmann tetrad in Leopard syndrome. Pediatr Neurol Nov-Dec 1989; $\underline{5}: 391-392)$. 